\title{
Medical abortion
}

\author{
Safe, effective, and legal in Britain
}

With the clinical licensing of oral mifepristone (RU 486) in combination with vaginal gemeprost, medical abortion is now legal in Britain in pregnancies up to and including the ninth week of amenorrhoea (seven weeks' gestation). The combination is effective and safe, ${ }^{1-3}$ with well over 100000 medical abortions performed. In licensing mifepristone Britain follows France, where one in five women seeking termination now opt for medical abortion, and China.

Provider units in the NHS and the private sector are currently preparing to offer medical abortion, and purchasing authorities are likely to seek provision of this service. Roussell, mifepristone's manufacturers, arranged a series of nationwide seminars for interested professionals, and attendance at these has been a prerequisite for the drug's supply.

The abortion that follows mifepristone and gemeprost resembles a spontaneous miscarriage and will be complete, requiring no surgical intervention, in $95 \%$ of cases. ${ }^{23}$ Most women find the process uncomfortable but acceptable; the majority of those who have experienced both medical and surgical abortion prefer the medical option. ${ }^{4}$ Complications and short term psychiatric morbidity seem similar for medical and surgical abortions. ${ }^{+5}$

The licence covers the combination treatment when used within nine weeks'amenorrhoea in women under 35 years old who are fit and no more than light smokers. ${ }^{6}$ These strict conditions have probably been imposed because of the solitary death in France of a 31 year old who had had 13 pregnancies and who smoked 40 cigarettes a day. (She suffered a cardiovascular collapse in association with intramuscular sulprostone, a prostaglandin that has since been superseded.)

Early accurate diagnosis of intrauterine pregnancy is a condition of the licence and is vital if women are to present and be counselled within the 28 to 63 day window. Monoclonal pregnancy tests are reliably positive at the time of the missed period, and direct telephone referral to a district coordinating office may reduce delays by weeks rather than days. Ultrasonography can confirm the site and viability of the pregnancy during the sixth week and is a useful adjunct to the full clinical assessment.

Prompt referral will allow time for counselling and decisions - including the often neglected option of continuing with the pregnancy. This will avoid the need for rushed decisions, which is otherwise a justifiable criticism of this technique. It is essential that the choice for the consumer must be a real one, with both surgical and medical abortion available in the same unit. In France about half of those attending centres offering medical abortion who fulfil the licence criteria choose medical abortion. Two doctors need to support the woman's request under the terms of the Abortion Act, and the abortion must commence at and be monitored through an NHS unit or licensed premises.

The treatment entails supervised oral administration of $600 \mathrm{mg}$ mifepristone, preferably on a later day than the counselling appointment - to allow time for reflection. Prescription controls allow each tablet to be traced by the company through to the individual patient. Consent must be obtained, including consent for surgical intervention should medical termination be incomplete or the tablets not be absorbed (which may result from the nausea and vomiting of early pregnancy). Two hours after taking the tablet the woman is discharged home and readmitted $36-48$ hours later. Non-steroidal anti-inflammatory analgesics should be avoided as they interfere with the metabolism of prostaglandins.

A contact telephone number must be provided, with easy access to medical advice and clinical assessment. About half of patients will have some bleeding, and 3\% will abort before their readmission. Clinical care is best provided in a day unit staffed by nurses committed to this option, with medical back up available at short notice.

A pessary containing gemeprost $1 \mathrm{mg}$ is inserted into the vagina, preferably early in the morning. Ninety per cent of women will have aborted within six hours and $96 \%$ within eight hours. ${ }^{23}$ Although success is achieved in $99 \%$ of women by 24 hours, some may wish to opt for a surgical evacuation after eight hours, possibly at the end of an afternoon surgical list. Narcotic analgesics are needed by one third, most commonly by nulliparous women of later gestation. Less than $1 \%$ of women will have bleeding severe enough to warrant urgent surgical evacuation of the products of conception. Between one quarter and one third of women will experience diarrhoea or vomiting, which are side effects of prostaglandin. ${ }^{2}$

Ideally the abortion should be confirmed by identifying the conceptus, as in a surgical evacuation. If not, a further diagnostic test is required, such as ultrasonography. Over half the women will be able to return home by that afternoon. Contraceptive advice must be given. ${ }^{7}$

A fourth visit is made seven to 12 days after the abortion, though $3 \%$ of women will need a surgical evacuation for continued bleeding before then. The clinical picture is 
probably more reliable than ultrasonography in deciding who needs an evacuation of retained products: of 507 scans performed seven days after a medical abortion, 47 suggested retained products, but only nine of these women needed admission. $^{2}$

Mifepristone also seems effective in reducing the physical and emotional trauma of abortion in the second trimester, whether performed under the Abortion $\mathrm{Act}^{8}$ or for intrauterine death. ${ }^{9}$ It greatly reduces the time that elapses between the administration of vaginal gemeprost and abortion, making this possible within a day care setting.

Both the Royal College of Obstetricians and Gynaecologists and the Birth Control Trust have recently published their recommendations for unplanned pregnancies. ${ }^{70}$ The scarcity of theatre time is one of the important limiting factors in

1 Guillebaud J. Medical termination of pregnancy. BMF 1990;303:352-4.

2 UK Multicentre Trial. The efficacy and tolerance of mifepristone and prostaglandin in first trimester termination of pregnancy. Br f Obstet Gynaecol 1990;97:480-6.

3 Silvestre L, Dubois C, Renault M, Rezvani Y, Baulieu E, Ulmann A. Voluntary interruption of pregnancy with mifepristone (RU486) and a prostaglandin analogue. Lancet 1990;335: $645-8$.

4 Urqhart DR, Templeton AA. Psychiatric morbidity and acceptability following medical and surgical methods of induced abortion. Br f Obstet Gynaecol 1991; 98: 396-9.

5 Mackenzie IZ. The potential effects on NHS resources. In: Williams C, ed. The abortion pill. London: Birth Control Trust, 1990:41-7. providing an adequate abortion service, and, on average, medical abortions require substantially less time in theatre than surgical abortions. They are, however, more labour intensive, and although financial savings have been suggested, no rigorous economic evaluation has yet been performed.

MICHAEL HEARD

Senior Registrar

Department of Obstetrics and Gynaecology,

University College Hospital,

London WC1E 6AU

JOHN GUILLEBAUD

Medical Director,

Margaret Pyke Centre for Study and

Training in Family Planning,

London W1V 5TW

6 Roussel Laboratories. Data Sheet. Mifegyne tablets (mifepristone). Uxbridge, Middlesex: Roussel Laboratories, 1991

Birth Control Trust. NHS abortion and RU486/gemeprost. London: Birth Control Trust, 1991.

8 Hill NCW, Selinger M, Ferguson J, Lopez-Bernal A, Mackenzie IZ. The physiological and clinical affects of progesterone inhibition with mifepristone (RU486) in the second trimester. Br $\mathcal{F}$ Obstet Gynaecol 1990;97:487-92.

9 Cabrol D, Dubois C, Cronje H, Gennet JM, Guillot M, Maria B, et al. Induction of labour with mifepristone (RU486) in intrauterine death. Am f Obstet Gynecol 1990; 163: 540-1.

10 Working Party of the Royal College of Obstetricians and Gynaecologists. Report on unplanned pregnancy. London: RCOG, 1991.

\section{Hepatocellular carcinoma}

\section{Referral to a specialist centre is recommended}

Worldwide, hepatocellular carcinoma is responsible for at least one million deaths a year. ${ }^{1}$ More than half the patients with this tumour also have cirrhosis of the liver, and this makes the treatment more difficult. ${ }^{2}$ Most patients die untreated.

The outlook, however, could be improved. Early diagnosis offers the best chance of cure, and careful monitoring of patients with chronic liver disease, particularly cirrhosis, has made it possible to detect hepatocellular carcinomas at an early stage. ${ }^{34}$ The five year survival rate after surgical resection varies from $30 \%$ to $50 \%$, depending on the proportion of asymptomatic or small cancers and how many patients also have cirrhosis. ${ }^{3-5}$ Tang et al found that five year survival after resection was $85 \%$ for those with tumours less than $2 \mathrm{~cm}$ in diameter but only $60 \%$ for those with tumours of $4 \cdot 1-5 \cdot 0 \mathrm{~cm} .{ }^{6}$ The prognosis of patients with a solitary, small hepatocellular carcinoma in a healthy liver is good. ${ }^{7}$ Nagasue et al showed that in patients with hepatocellular carcinoma the four year survival rate was $80 \%$ in those without cirrhosis but $35 \%$ in those with cirrhosis. ${ }^{8}$

Untreated, the natural course of patients with even small liver cancers (less than $3 \mathrm{~cm}$ in diameter and asymptomatic) is poor. A study of 22 patients found that only three were alive three years after the tumour was detected. ${ }^{9}$ Among 12 patients who died the cause of death in five was variceal bleeding rather than advanced cancer. In the seven patients who died of massive cancer the tumours grew rapidly, and invasion of the portal vein by cancer was shown by ultrasonography in four.

The results of any treatment should be set against this figure of $13 \%$ survival at three years. For example, surgical resection of cancers of the liver as small as $3 \mathrm{~cm}$ gave a four year survival rate of $59 \%$ - a figure that has validated the operation. ${ }^{10}$ Small and encapsulated hepatocellular carcinoma is nowadays being both diagnosed and treated throughout the world. ${ }^{511}$
In the 1980s liver surgery became both safer and more widely practised-mainly because of technical advances. ${ }^{12} 13$ The treatment of patients with hepatocellular carcinoma and cirrhosis remains difficult to decide. Those with adequate biochemical liver function are usually good candidates for surgery. ${ }^{1+}$ Perioperative mortality and morbidity seem to depend on the volume of operative blood loss and the extent of the resection.

Follow up studies have shown that the main hazard after resection of hepatocellular carcinoma is intrahepatic recurrence; rates may be $50 \%$ or more within one to two years of operation. ${ }^{15-17}$ Recurrent tumours (and primary tumours that cannot be resected) may be treated by transcatheter arterial embolisation, ${ }^{18}$ targeted chemotherapy, ${ }^{19}$ or percutaneous injection of alcohol. ${ }^{2021}$ These treatments have improved the prognosis in patients with recurrent disease. ${ }^{22}$ Patients with hepatocellular carcinoma less than $3 \mathrm{~cm}$ treated by ultrasonographically guided percutaneous injection of alcohol at one Japanese unit had a five year survival rate of $48 \% .{ }^{23}$

The results of liver transplantation for hepatocellular carcinoma have been disappointing, ${ }^{24-26}$ but those for end stage cirrhosis with incidental malignancy are much better. ${ }^{24}$ The best advice for a patient with the disease is to be referred to a specialist centre staffed by surgeons and physicians who are familiar with hepatocellular carcinoma.

Assistant Professor,

Second Department of Surgery, School of Medicine,

University of Occupational and Environmental Health,

Kitakyushu 807,

Japan

Rustgi VK. Epidemiology of hepatocellular carcinoma. Ann Intern Med 1988;108:390-1.

2 Kew MC, Popper H. Relationship between hepatocellular carcinoma and cirrhosis. Semin Liver Dis 1984;4:136-46.

3 Lee CS, Sung JL, Hwang LY, Sheu JC, Chen DS, Lin TY, et al. Surgical treatment of 109 patients with symptomatic and asymptomatic hepatocellular carcinoma. Surgery 1986;99:481-90. 